\title{
El problema teoría-práctica en los modelos de formación del profesorado: Una mirada psicológica
}

\author{
The theory-practice problem in teacher education models: \\ a psychological perspective
}

\author{
Marc Clarà \\ Universitat de Lleida, España. \\ marc.clara@gmail.com
}

\begin{abstract}
RESUMEN
Este artículo sugiere que algunas discrepancias entre los distintos modelos de formación del profesorado están relacionadas con distintas formas de definir el problema teoría-práctica. Se propone tres formas de definir el problema: como un problema de transferencia entre contextos; como un problema de relación entre lo explícito y lo implícito; como un problema de relación entre lo proposicional y lo situacional. Seguidamente, se relacionan estas distintas visiones del problema con cuatro modelos de formación del profesorado: basado en la escuela (SBTE), basado en la investigación (RBTE), realístico (RTE), y basado en prácticas centrales (CPTE). Se propone que la SBTE y la RBTE definen el problema en términos de transferencia entre contextos, el RTE lo define a partir del eje explícito-implícito, y el CPTE lo define mediante del eje proposicional-situacional. Se postula que ninguno de los modelos examinados resuelve la cuestión pero que todos presentan algunos elementos que pueden significar avances.
\end{abstract}

Palabras clave: formación del profesorado; relación teoría-práctica; conocimiento del profesor.

\begin{abstract}
This article suggests that some discrepancies between the different models of teacher education are related with different ways of defining the theory-practice problem. The article proposes three ways of defining the problem: as a problem of transference between contexts; as a problem of relation between the explicit and the implicit; as a problem of relation between the propositional and the situational. These different views of the problem are then linked to four models of teacher education: school-based (SBTE), research-based (RBTE), realistic (RTE), and core practices (CPTE). It is proposed that SBTE and RBTE define the problem in terms of transfer between contexts, the RTE defines it from the explicit-implicit axis, and the CPTE defines it from the propositionalsituational axis. It is argued that none of the models examined resolves the issue, but that they all contain some elements that may represent advances.
\end{abstract}

Key words: teacher education; theory-practice relationship; teacher knowledge. 


\section{INTRODUCCIÓN}

La organización de la formación inicial del profesorado se encuentra en continua discusión desde hace ya varias décadas. A grandes rasgos, esta discusión se podría describir como un vaivén entre modelos que abogan por poner más énfasis en la formación académica en contextos universitarios y, modelos que defienden que hay que basar la formación de los futuros profesores en los contextos de práctica docente. En parte, esta discusión versa sobre diferencias de tipo pedagógico con relación a cómo imaginamos, como sociedad, la figura y el trabajo del profesor, así como el rol y la función de la escuela (Aspfors y Eklund, 2017; Zeichner, 2014). Sin embargo, entre los distintos modelos hay también una discusión de fondo sobre cómo hay que resolver el problema de la falta de articulación entre la teoría y la práctica o, en otras palabras, cómo hay que organizar la formación inicial para maximizar su impacto en el posterior desempeño profesional de los profesores (Admiraal, Buijs, Claessens, Honing y Karkdijk, 2017; Cochran-Smith y Lytle, 1999; Korthagen, 2017).

Este artículo propone que los diferentes modelos de formación inicial del profesorado responden a visiones esencialmente distintas sobre cuál es, en qué consiste, y porque ocurre este problema de articulación entre teoría y práctica; por esta razón, porque parten de planteamientos del problema fundamentalmente distintos, proponen también formas distintas de organizar la formación del profesorado. El objetivo de este artículo es clarificar la relación entre los distintos modelos de formación del profesorado y las distintas formas de explicar psicológicamente el problema de la articulación entre teoría y práctica. Como se intentará mostrar, añadir este estrato psicológico a la discusión puede profundizar la comprensión de las propuestas organizativas de cada modelo, permitiendo identificar limitaciones y líneas de mejora para la articulación teoría-práctica en los distintos modelos.

\section{LA RELACIÓN ENTRE TEORÍA Y PRÁCTICA: UN PROBLEMA PSICOLÓGICO}

Durante la mayor parte del siglo XX, la relación teoría-práctica se consideró a la luz de una serie de supuestos que algunos autores han denominado "ideología de la racionalidad" (Lave, 1991), o "racionalidad técnica" (Schön, 1998). En primer lugar, se asumía que ciertos aprendizajes necesarios para una práctica competente tenían que ser aprendidos en un contexto ad-hoc, generado expresamente para realizar dichos aprendizajes, y diferente del contexto de práctica. En segundo lugar, se asumía que en estos contextos ad-hoc, el aprendizaje que debía realizarse era fundamentalmente de tipo proposicional, explícito y general. Es decir, aprendizaje verbalizable, consciente, y generado mediante investigación académica. En tercer lugar, se asumía que una vez estas proposiciones habían sido aprendidas en los contextos ad-hoc eran transferidas a los contextos de práctica. Finalmente, en cuarto lugar, se asumía que, una vez en el contexto de práctica, estas proposiciones guiaban la toma de decisiones y la acción del sujeto.

A partir de la década de 1980, este conjunto de supuestos empezó a ser seriamente cuestionado por la evidencia empírica. Desde diferentes perspectivas se fue mostrando evidencia de que los supuestos que subyacen la racionalidad técnica son, al menos en parte, falsos. Sin embargo, treinta años después, aún no existe unanimidad sobre qué supuestos son falsos y qué supuestos son válidos; no existe acuerdo sobre qué es lo que falla en la racionalidad técnica, y en consecuencia hay varias explicaciones alternativas sobre cómo 
funciona realmente la relación teoría-práctica. En otras palabras, el problema de la relación entre teoría y práctica consiste, fundamentalmente, en que no sabemos aún dónde está el problema.

Hay, eso sí, algunas hipótesis al respecto. Por un lado, algunos autores proponen que el tercer supuesto de la racionalidad técnica, el de la transferencia entre contextos, es falso. Otros proponen que el supuesto que es falso es el cuarto, el que asume que las proposiciones explícitas guían la acción. Entre los que niegan el cuarto supuesto, hay algunos que señalan que lo que es falso es que las proposiciones que guían la práctica sean explícitas; sugieren que son proposiciones implícitas las que dirigen la acción. Otros señalan que el error está en asumir que la práctica está guiada por proposiciones; sugieren que la acción está dirigida por conocimiento no proposicional. La cuestión está aún en el aire y el problema psicológico sigue sin resolver, de modo que las tres formulaciones del problema coexisten y condicionan la organización de la formación del profesorado, dando lugar a propuestas de formación claramente distintas según si el problema teoría-práctica se define de un modo o de otro. En los próximos apartados, intentaré exponer estas tres alternativas psicológicas y su impacto en las principales propuestas actuales sobre formación del profesorado.

\section{HIPÓTESIS 1: LA TRANSFERENCIA DE CONOCIMIENTO ENTRE CONTEXTOS NO ES POSIBLE}

Una forma de explicar el problema de la relación teoría-práctica consiste en postular que la transferencia de conocimiento desde un contexto a otro diferente no es posible. Por lo tanto, se postula que el tercer supuesto de la racionalidad técnica, asumido durante largo tiempo, es falso: la transferencia no existe. Negando el tercer supuesto, los dos primeros supuestos dejan también de tener sentido: si la transferencia entre contextos no es posible, ¿qué sentido tiene crear un contexto ad-hoc para el aprendizaje, si este aprendizaje no podrá ser transferido al contexto de práctica?

Esta hipótesis fue puesta de relieve por un conjunto de investigaciones que, aunque diferentes entre ellas, pueden ser agrupadas bajo el término de "cognición situada" (Hutchins, 1995; Lave, 1991; Nardi, 1996; Nunes-Carraher, Carraher y Schliemann, 1985; Scribner, 1984). Básicamente, estas investigaciones mostraron que los procesos cognitivos se llevan a cabo mediante los artefactos concretos que están disponibles en sus contextos específicos. En otras palabras, la cognición no es un fenómeno puramente interno, sino que se distribuye en el contexto: los artefactos presentes y disponibles en un contexto son instrumentos cognitivos son medios para la cognición. Nunes-Carraher et al. (1985), por ejemplo, estudiaron niños vendedores de cocos en Recife (Brasil), y mostraron que la forma en que hacían los cálculos en el mercado difería mucho de cómo hacían cálculos similares en contexto escolar. Algo similar documentó también Lave (1991), estudiando cómo las personas calculaban su compra en el supermercado, y como personas que participaban en un programa de dieta calculaban las cantidades de los alimentos.

Si se acepta que un proceso cognitivo está constituido por los artefactos distribuidos en el contexto, puede derivarse la idea de que cuando el contexto cambia, cuando los artefactos disponibles son diferentes, aquel proceso cognitivo deja de ser posible, porque sus elementos constitutivos desaparecen. Por lo tanto, un proceso cognitivo no puede ser transferido de un contexto a otro diferente; si el contexto cambia, la cognición será necesariamente otra 
distinta. Así pueden explicarse las diferencias en la forma de hacer cálculos matemáticos entre la escuela, el mercado de Recife, el supermercado, y durante la preparación de las comidas. Y así puede explicarse también por qué aquello que los maestros aprenden en la universidad es raramente movilizado en las escuelas donde trabajan.

\subsection{LA TRANSFERENCIA COMO CLAVE DEL PROBLEMA: PROPUESTAS DE FORMACIÓN INICIAL DEL PROFESORADO}

La hipótesis de que el problema teoría-práctica radica en la imposibilidad de transferencia entre contextos ha dado lugar, a mi modo de ver, a dos propuestas de formación del profesorado que parecen, por lo demás, absolutamente opuestas entre ellas. Una de ellas se ha denominado a veces "school-based teacher education" (SBTE) (formación del profesorado basado en la escuela); la otra, "research-based teacher education" (RBTE) (formación del profesorado basado en la investigación).

La propuesta lanzada por la SBTE es simple. Si la transferencia no es posible, no tiene mucho sentido situar el aprendizaje en un contexto diferente del contexto en el que se tiene que usar. El aprendizaje, por lo tanto, tiene que realizarse directamente en el contexto de práctica, lo que significa aumentar el tiempo de formación en la escuela y reducir el tiempo de formación en la universidad (ten Dam y Blon, 2006; van Velzen y Volman, 2009; Zeichner, 2012). Esta propuesta, obviamente, genera algunas preguntas y preocupaciones. Quizás la más evidente es el peligro de la reproducción acrítica por parte de los estudiantes de lo que algunos autores han llamado "pedagogías mediocres" (Stones, 1992, citado en Buitink, 2009, p. 118), así como un posible estancamiento de las prácticas docentes en las escuelas, ya que un énfasis excesivo en la reproducción podría perjudicar los procesos de innovación y cambio necesarios en las escuelas de nuestro tiempo (Lawson, Norwich y Nash, 2013). En este sentido, Cochran-Smith y Lytle (1999) propusieron que la SBTE tiene que plantearse desde una "postura de indagación" (inquiry stance), de forma que, en la escuela, el estudiante debe orientarse a generar conocimiento local mediante procesos de investigación-acción en el marco de la comunidad; de esta forma, el peligro de una reproducción acrítica de prácticas en la SBTE podría ser atenuado. En la propuesta de Cochran-Smith y Lytle es importante enfatizar, primero, que la indagación se propone en la escuela y desde la escuela; segundo, que el proceso de indagación propuesto por estas autoras se diferencia claramente de un proceso de investigación de tipo académico; y tercero, que el conocimiento que busca generarse es de tipo local, es decir, conocimiento del contexto concreto en el que se está trabajando y no se busca una generalización a otros contextos. Otra tensión evidente que genera el modelo del SBTE es que la universidad como contexto formativo y la investigación académica como fuente de conocimiento parecen dejar de tener sentido. Una forma de abordar esta tensión ha sido proponer que los profesionales de la universidad participen en los procesos de indagación que los estudiantes, juntamente con la comunidad escolar, llevan a cabo en la escuela, configurando así lo que se ha llamado un "tercer espacio", sustentado por convenios entre escuela y universidad (Jackson y Burch, 2018; Lillejord y Borte, 2016; Zeichner, 2010).

Una alternativa opuesta para resolver el problema de la transferencia es la RBTE. Desde esta perspectiva, la idea básica es construir un contexto de práctica que incluya tanto la universidad como la escuela; es decir, pasar de una situación en la que lo académico y lo escolar son dos contextos separados a una nueva situación en la que sean dos aspectos 
complementarios de un único contexto de práctica (Admiraal et al., 2017; Toom et al., 2008). Esto, claro está, significa expandir el concepto de "práctica educativa" para incluir en este las tareas de investigación académica (Maaranen y Krokfors, 2008; Smith, 2015). De esta forma, el problema de la transferencia podría ser resuelto, porque la separación entre contextos de práctica se diluye y, por lo tanto, no hay transferencia que hacer: el conocimiento es generado académicamente en el mismo contexto (complejo y multi-institucional) en el que tendrá que ser usado. Este planteamiento implica que los profesores tienen que construirse como científicos de la educación, como académicos. Esto supone aumentar sustancialmente la formación científica de los futuros profesores, incluyendo formación en métodos de investigación académica, lo que implica un aumento de las horas de formación en la universidad y un alargamiento de los grados de educación (normalmente, hasta a nivel de Máster - 5 años, incluyendo una tesis final de investigación) (Toom et al., 2010). Este camino es, como se puede ver, casi opuesto al que se toma desde la SBTE; opuesto en el sentido de que mientras la SBTE reduce el tiempo de formación en la universidad, la RBTE lo expande. Sin embargo, debe aclararse que el RBTE no niega la importancia de que una parte de la formación se haga también en la escuela. La RBTE presenta, en principio, algunas ventajas en relación con la SBTE: atribuye un rol importante a la investigación académica y al conocimiento proposicional general y conlleva un incremento del prestigio social de los profesores (Aspfords y Eklund, 2017). Sin embargo, se trata de un modelo difícil de implementar, que demanda un proceso largo y sostenido en el tiempo. Además, no está claro que este modelo pueda resolver efectivamente la articulación teoría-práctica. Finlandia ha mantenido este modelo durante 4 décadas (Tirri, 2014) y la evidencia sobre el éxito en la cuestión teoría-práctica es contradictoria: aunque hay cierta evidencia de que los estudiantes asumen una postura reflexiva y crítica, atribuible a la formación científica y en investigación, es difícil de detectar un impacto directo de la formación basada en investigación, y de la investigación misma y sus resultados, en la práctica de los profesores (Aspfors y Eklund, 2017; Hansén, Forsman, Aspfors y Bendtsen, 2012; Maaranen y Krokfors, 2008; Puustinen, Säntti, Koski y Tammi, 2018). Hacen falta más experiencias de largo recorrido (Noruega ha hecho una apuesta decidida en esta dirección) para dilucidar si, además de los claros beneficios que este modelo conlleva, especialmente los derivados del incremento del prestigio social de los profesores, el RBTE puede suponer también una solución para el problema teoría-práctica.

\section{LAS PROPOSICIONES EXPLÍCITAS NO GUÍAN LA PRÁCTICA, LO HACEN LAS PROPOSICIONES IMPLÍCITAS}

Una forma distinta de explicar el problema teoría-práctica radica en plantearlo en términos de relación conocimiento-acción. Desde este punto de vista, se ha propuesto la hipótesis de que la acción viene determinada por ideas o conocimientos de los que el sujeto no es consciente, que son implícitos. Además, estos conocimientos implícitos están desconectados psicológicamente de los conocimientos o ideas de las que el sujeto sí que es consciente (explícitos). Por lo tanto, se puede dar el caso que una persona mantenga una idea de forma consciente, pero que implícitamente, sin darse cuenta, tenga otra idea contradictoria; es esta última idea la que guía la acción. Esto explicaría la distancia que a menudo hay entre lo que se dice y lo que se hace. Nótese que este planteamiento no niega 
la transferencia: la proposición explícita efectivamente se transfiere, el problema es que no es ésta la que guía la acción, sino otras proposiciones de las que el sujeto no es consciente.

Esta hipótesis ha sido propuesta fundamentalmente por aproximaciones cognitivas que han planteado lo que se puede llamar "modelos de doble proceso." Se han propuesto varios de estos modelos; tres de los más influyentes son los propuestos por Fodor (1983), Epstein (2003) y Kahneman (2003). Aunque son sensiblemente diferentes entre ellos, los tres modelos tienen importantes elementos en común. Básicamente, estos modelos proponen que el procesamiento de la información tiene lugar en dos sistemas computacionales más o menos independientes. Uno de ellos -Fodor lo llama "módulo", Epstein lo llama "sistema experiencial" y Kahneman lo llama "sistema 1"- se caracteriza por ser un sistema de procesamiento rápido, del orden de los milisegundos, e implícito, no es controlado conscientemente por el individuo. Además, se trata de un sistema encapsulado: esto significa que procesa sólo la información disponible en este mismo sistema, la información disponible en otros sistemas no interviene en la computación. Este sistema es responsable de la toma de decisiones. El otro sistema computacional -llamado "sistema central" (Fodor), "sistema racional" (Epstein), o "sistema 2" (Kahneman)- es un sistema de procesamiento lento, que puede ser controlado conscientemente por el individuo y que no es encapsulado.

Por lo tanto, según esos modelos, la información resultante o disponible en el sistema 1 puede formar parte de computaciones en el sistema 2, pero no a la inversa. En otras palabras, el sistema 1, mediante el cual tomamos las decisiones, es ciego a los procesos de razonamiento y aprendizaje consciente que tienen lugar en el sistema 2. Esta es la razón de la disgregación entre teoría y práctica, entre conocimiento explícito y acción. Los tres modelos conducen a la conclusión de que, debido a la arquitectura computacional de la mente, es imposible que el conocimiento explícito penetre en la toma de decisiones. Como mucho, Epstein y Kahneman sugieren que, dado que el sistema 2 puede computar los outputs del sistema 1, el conocimiento explícito puede servir, cuando hay tiempo suficiente, para evaluar la decisión a posteriori.

\subsection{EL EJE IMPLÍCITO-EXPLÍCITO COMO CLAVE DEL PROBLEMA: PROPUESTAS DE FORMACIÓN INICIAL DEL PROFESORADO}

Los modelos de doble proceso implican aceptar una división insalvable entre conocimiento explícito y acción. Sin embargo, algunos autores han intentado abrir la posibilidad de que superar esta división entre sistemas computacionales pudiera ser posible; esta posibilidad ha tenido un impacto relevante en el campo de la formación del profesorado. En un intento de modificar el planteamiento de Fodor para hacerlo compatible con una aproximación evolutiva, Karmiloff-Smith (1992) propuso que las representaciones pueden re-describirse en formatos diferentes. Específicamente, sugirió que representaciones implícitas pueden redescribirse gradualmente en representaciones cada vez más explícitas. Esta idea ha impregnado las propuestas de algunos autores preocupados por el problema teoría-práctica en la formación del profesorado, como Pozo (Pozo, Scheuer, Mateos y Pérez Echevarría, 2006; Pozo et al., 2010) o Korthagen (2001, 2010a). Básicamente, estos autores proponen que las representaciones implícitas en el sistema 1 pueden ser progresivamente redescritas en formatos más explícitos, hasta llegar a ser equivalentes, en cuanto a formato, a las propias del sistema 2. Una vez explicitadas, pueden entrar en contacto con representaciones explícitas generadas mediante investigación académica y aprendidas de forma consciente, 
y pueden ser así modificadas en el sistema 2. Luego, pueden ser de nuevo redescritas en formatos cada vez más implícitos (lo que Korthagen [2010b] llama "reducción de nivel”, y Pozo [2017] “implicitación”), para poder ser computadas de nuevo por el sistema 1.

Algunas propuestas de formación del profesorado -quizás la más relevante sea la que se ha llamado "Realistic Teacher Education" (formación del profesorado realística) (RTE) - se han basado en esta idea de redescripción representacional. Básicamente, el modelo implica, para empezar, un cierto equilibrio entre el tiempo de formación en la escuela y el tiempo de formación en la universidad. Lo que es especialmente distintivo del modelo es que la formación en la universidad se construye a partir de la experiencia de los estudiantes en la escuela: se intenta llevar a cabo un proceso de explicitación progresiva de las representaciones del estudiante, implícitas en su acción, a través de un ciclo de reflexión denominado ALACT (Action, Looking Back, Awareness, Creating an alternative, Trial/ Action) y el ciclo vuelve a empezar. Este modelo ha cosechado algunos éxitos importantes: en Holanda, donde esta propuesta ha sido implantada desde hace ya algunas décadas, las evaluaciones del modelo, tanto por parte de expertos externos, como de los propios estudiantes, como de los directores de los centros en los que van a trabajar los graduados, han sido muy superiores a las recibidas por otros programas en el contexto holandés (Korthagen, 2010a). Sin embargo, la evidencia levanta algunas dudas sobre si este modelo puede realmente suponer una solución al problema teoría-práctica. En particular, pueden existir dudas de que la idea de redescripción representacional, como clave para dicha solución, sea correcta. La evidencia efectivamente muestra que, a partir de la experiencia en la escuela, los estudiantes pueden explicitar ideas y creencias que estaban implícitas en su acción. No obstante, por un lado, se ha demostrado muy complicado que las ideas inicialmente implícitas lleguen a redescribirse en ideas lo suficientemente generales y explícitas como para poder dialogar con las teorías académicas (Korthagen, 2010a). Por otro lado, las ideas implícitas, aunque sean explicitadas, parecen ser extremadamente resistentes al cambio (Park, Hewson, Lemberger y Marion, 2010; Pozo, 2007; Tillema y Knoll, 1997; Duit y Treagust, 2003). Delante de esta evidencia, Korthagen (2017) propone que el tipo de conocimiento explícito que deben desarrollar los profesores es de tipo local, lo que él llama "phronesis", "teoría con t minúscula" (Korthagen y Kessels, 1999), o "teorías personales", una forma de conocimiento similar a la que Cochran-Smith y Lytle (1999) persiguen generar en su versión de SBTE (discutido anteriormente). Sin embargo, de acuerdo con los modelos de doble proceso, cabe preguntarse si este conocimiento explícito local, una vez implicitado, guía la acción (y por lo tanto el supuesto de la redescripción es correcto), o si la relación es la inversa, es decir, si este conocimiento local se genera a posteriori para legitimar o justificar la acción, pero no interviene en la toma de decisión (tal y como predicen los modelos de doble proceso si no se asume la redescripción).

\section{HIPÓTESIS 3: LA ACCIÓN NO ESTÁ GUIADA POR PROPOSICIONES, NI EXPLÍCITAS NI IMPLÍCITAS, SINO QUE ESTÁ GUIADA POR REPRESENTACIONES NO PROPOSICIONALES}

Una tercera forma de plantear el problema teoría-práctica es, de nuevo, negar la validez del supuesto 4 -según el cual el conocimiento proposicional explícito guía la acción-, pero por una razón diferente: según este planteamiento, la cuestión no está en si el conocimiento es 
explícito o implícito; el problema radica en que la influencia del conocimiento en la acción no tiene lugar según la lógica proposicional. En otras palabras, no son proposiciones lo que guían la toma de decisión, sino otro tipo de conocimiento.

Tanto la racionalidad técnica como las hipótesis alternativas anteriores asumen que el pensamiento y la toma de decisión obedecen a una lógica proposicional. Este tipo de lógica postula que se llega a conclusiones (o a decisiones) a partir de un conjunto de relaciones lógicas entre proposiciones (o premisas). Por ejemplo, una secuencia lógica proposicional puede tomar la siguiente forma:

- Si A, entonces B

$-\mathrm{A}$

$\rightarrow \mathrm{B}$

Podemos aplicar esta estructura a un ejemplo relevante para la formación del profesorado:

- La interacción dialógica en clase favorece la intersubjetividad

- Interacción dialógica en clase

$\rightarrow$ Intersubjetividad

Si se asume que se busca generar intersubjetividad, este razonamiento de tipo proposicional llevaría al profesor a organizar la clase en forma de interacción dialógica.

Johnson-Laird (1983) propuso que la asunción de que el razonamiento responde a esta lógica proposicional es falsa. Este autor observó que, de forma sistemática y generalizada, los humanos cometemos errores de razonamiento que son difíciles de explicar si se asume que razonamos proposicionalmente. Por ejemplo, delante del silogismo formado por las premisas,

- Todos los B son A

- Ningún C es B

hay una tendencia generalizada a derivar la conclusión:

$\rightarrow$ Ningún C es A

lo que es incorrecto. Este tipo de error, Johnson-Laird lo llama "figural effect". La explicación que el autor da al hecho que se dé este tipo de errores generalizados es que no razonamos mediante lógica proposicional, sino que lo hacemos mediante la construcción de modelos mentales. Básicamente, Johnson-Laird propone que el proceso funciona del siguiente modo: imaginamos una situación concreta en la que la primera premisa (todos los B son A) sea válida, por ejemplo:

$$
\begin{aligned}
& \mathrm{B}=\mathrm{A} \\
& \mathrm{B}=\mathrm{A} \\
& \mathrm{B}=\mathrm{A} \\
& \mathrm{B}=\mathrm{A}
\end{aligned}
$$


A continuación, incorporamos la segunda premisa (ningún C es B) en esta situación, de forma que ésta también sea válida, por ejemplo:

\begin{tabular}{|l|}
$\mathrm{B}=\mathrm{A}$ \\
$\mathrm{B}=\mathrm{A}$ \\
$\mathrm{B}=\mathrm{A}$ \\
$\mathrm{B}=\mathrm{A}$ \\
$\mathrm{C}^{\mathrm{C}}$ \\
$\mathrm{C}$ \\
$\mathrm{C}$
\end{tabular}

Una vez construido el modelo, sacamos conclusiones: ningún $\mathrm{C}$ es A. Esto explicaría el "figural effect". Johnson-Laird propone que, una vez hemos construido un modelo, si hay tiempo, empezamos a buscar contra-ejemplos: modelos alternativos en los que las dos premisas sean también válidas. La construcción del modelo inicial es rápida e implícita; la búsqueda de contra-ejemplos es un proceso consciente y que demanda tiempo. Estos dos momentos del proceso equivalen pues al razonamiento implícito y explícito. Sin embargo, a diferencia de los modelos de doble proceso, esta propuesta no los considera procesos de distinta naturaleza, que tienen lugar mediante dos sistemas computacionales diferentes; en esta propuesta son simplemente dos momentos de un mismo proceso. Se reconoce por lo tanto el eje implícito-explícito, pero se considera que el razonamiento implícito y el explícito son de la misma naturaleza psicológica. Así, una vez formado el modelo inicial, si hay tiempo, se buscan modelos alternativos de forma consciente. Por ejemplo, un modelo alternativo es:

\begin{tabular}{|c|}
\hline $\mathrm{A}$ \\
$\mathrm{B}=\mathrm{A}$ \\
$\mathrm{B}=\mathrm{A}$ \\
$\mathrm{B}=\mathrm{A}$ \\
$\mathrm{A}=\mathrm{C}$ \\
$\mathrm{A}=\mathrm{C}$ \\
$\mathrm{C}$ \\
$\mathrm{C}$ \\
$\mathrm{C}$ \\
$\mathrm{C}$
\end{tabular}

donde las dos premisas son válidas, y en cambio la conclusión anterior no lo es. Aquella conclusión, por lo tanto, se percibe ahora como inválida y la conclusión válida que puede derivarse es que algunos $\mathrm{A}$ no son $\mathrm{C}$. 
Por lo tanto, la propuesta es que se construye una representación (un modelo) de la situación completa, y es a partir de esta representación global que sacamos conclusiones y tomamos decisiones. Como se ha comentado, este proceso de representar la situación puede ser rápido e inconsciente (implícito) en un primer momento, y lento y consciente cuando se buscan modelos alternativos (explícito). Uno de los autores que ha estudiado en profundidad la parte implícita del proceso es Klein; por lo que respecta a la búsqueda explícita de modelos alternativos, el trabajo de Schön ha tenido una gran influencia.

Klein $(2008,2015)$ propone que, en la toma de decisiones, el práctico dispone de lo que el autor denomina "repertorio", que consiste en un conjunto de modelos mentales previamente construidos para situaciones ya vividas en el pasado. Cuando el práctico se enfrenta a una situación, usa el repertorio para darle sentido, le aplica un modelo mental disponible en el repertorio, lo que le permite representar la situación y tomar decisiones de forma muy rápida e implícita. A este proceso, Klein (2008) lo llama "recognition-primed decision-making" (toma de decisiones basada en el reconocimiento). Schön (1987) hace una conceptualización bastante similar para el proceso implícito, que denomina "conocer en la acción":

cuando un profesional da sentido a una situación que él interpreta como singular, la ve como algo ya presente en su repertorio... Viendo esta situación como aquella otra, un práctico puede también comportarse en esta situación como en aquella otra (p. 71).

Sin embargo, Schön se interesa especialmente por las ocasiones en las que el repertorio (los modelos mentales previamente construidos) no se acomoda a la situación. En estos casos, se inicia un proceso explícito y lento de construcción de nuevos modelos o de reconstrucción de los que ya forman parte del repertorio -a este proceso explícito, Schön lo denomina "reflexión", basándose en algunos de los planteamientos de Dewey (Clarà, 2015). En este proceso de reflexión, en la construcción y re-construcción explícita de modelos, es donde el conocimiento proposicional explícito puede penetrar en estos modelos, y por lo tanto, influenciar la acción. Sin embargo, esta influencia no tiene lugar de forma lineal, como cabría esperarse según la lógica proposicional, sino de forma indirecta. Schön, por ejemplo, identifica un modo en la que la teoría influencia la acción, que él denomina "teoría abarcadora". El autor analiza una sesión entre un psicoanalista en formación (residente) y su supervisor, en la que los participantes intentan dar sentido (construir un modelo) a un caso que está llevando el psicoanalista. Se trata de una paciente que dejó la terapia con el residente y, después de unos meses, ha vuelto. El residente habla de por qué la mujer dejó la terapia:

R: Ella había decidido que no estaba obteniendo terapia en ninguna parte, y estuve algo de acuerdo en que los mismos temas estaban apareciendo una y otra vez -y sobre todo ese asunto de su fijación en la relación con el hombre que ella había estado viendo durante cuatro o cinco años por aquel tiempo-, y los avances por su parte, se correspondían con el abandono de él, y viceversa.

S: ¿De qué modo se quedó ella bloqueada contigo?, quiero decir, ¿fue en los mismos términos que el modo en el que se quedó bloqueada en la relación? (Schön, 1998, p. 106).

Schön hace notar que la pregunta del supervisor está fuertemente orientada por el concepto psicoanalítico de "transferencia". De esta forma, este concepto teórico orienta la mirada del supervisor hacia la información que puede ser relevante para la construcción del modelo, guiando y condicionando el proceso de reflexión. 
Johnson-Laird (1983) identifica otra forma en la que las proposiciones explícitas pueden penetrar en los modelos mentales: Las proposiciones pueden mapearse sobre los modelos, de forma que pueden ser pistas, puntos de referencia, a partir de los cuales se construye el modelo. Así, una proposición puede modificar una premisa del modelo o puede dar lugar a una nueva premisa. En cualquier caso, la proposición se integra, se funde, en el modelo mental. Dewey (1989) ofrece un ejemplo que puede ilustrar este fenómeno:

Al lavar vasos con agua caliente y jabón y colocarlos boca abajo en una bandeja, observé que aparecían burbujas en el exterior de la boca de los vasos y luego se metían adentro. ¿Por qué? La presencia de burbujas sugiere aire, que, según advertí, debía proceder del interior del vaso. Noté que el agua jabonosa de la bandeja impedía escapar el aire, salvo que quedara aprisionado en burbujas. Pero, ¿por qué salía el aire del vaso?... Debía expandirse. El aire se expande por aumento de calor o de presión, o por ambas cosas al mismo tiempo. ¿Podía haberse calentado el aire una vez estuviera el vaso fuera del agua caliente? ... Seguramente el aire del exterior se expandió a causa del calor del vaso, lo cual explica la aparición de burbujas en el exterior. Pero, ¿por qué entraban luego? El fría contrae. El vaso se enfriaba y también el aire que contenía. La tensión desaparecía, y, en consecuencia, las burbujas aparecían en el interior (p. 93, énfasis añadido).

En este ejemplo, el protagonista intenta construir un modelo que explique la situación. El modelo se va construyendo a partir de hechos observados (el jabón, la dinámica de las burbujas fuera-dentro del vaso) y también a partir de una proposición explícita (el aire se expande por aumento de calor y se contrae por el frío). La proposición y los hechos se mapean conjuntamente y en relación mutua, lo que permite construir un modelo satisfactorio para la situación (para más detalle, ver Clarà, 2014, 2015).

Así pues, esta hipótesis sugiere que las proposiciones explícitas pueden influenciar la acción, pero que esta influencia no es lineal ni directa como sería esperable según la lógica proposicional. La influencia de la teoría sobre la acción, en cambio, es difusa y a veces difícil de percibir, porque tiene lugar a través de su influencia en la construcción de los modelos mentales que, estos sí, guían la práctica.

\subsection{EL EJE PROPOSICIONAL-SITUACIONAL COMO CLAVE DEL PROBLEMA TEORÍA-PRÁCTICA: PROPUESTAS DE FORMACIÓN INICIAL DEL PROFESORADO}

La idea de que los modelos mentales, o el conocimiento de las situaciones, guían la práctica de los maestros (en lugar de hacerlo el conocimiento proposicional) tiene relación con una propuesta de formación del profesorado que recientemente ha emergido con fuerza y que a veces se ha denominado "Core Practices" (prácticas centrales) (CPTE) (Forzani, 2014; McDonald, Kazemi y Kavanagh, 2013). Básicamente, la CPTE propone que, dado que los maestros guían su práctica y toman decisiones a partir del conocimiento que tienen de las situaciones a las que se enfrentan, la formación del profesorado debe orientarse a construir este repertorio de conocimiento situacional. Para ello, es necesario, en primer lugar, identificar los tipos de situaciones que son centrales en la práctica de los maestros (lo que se ha llamado las Core Practices), y una vez identificadas, organizar el currículum alrededor del aprendizaje de estas. Desde dicha perspectiva, no se niega la posibilidad de transferencia 
entre contextos. En este sentido, la CPTE propone un cierto equilibrio entre formación en la escuela y formación en la universidad. Sin embargo, la formación en la universidad gira alrededor de las Core Practices, de forma que la articulación universidad-escuela se entiende como un continuum que permite progresar desde entornos de práctica simulada hacia entornos de práctica cada vez más auténtica (Grossman et al., 2009). Se entiende, además, que el repertorio debe construirse de forma activa y consciente -no basta con vivir la situación, es necesario un proceso explícito que se ha denominado "descomposición de la práctica"- (Grossman et al., 2009; Peercy y Troyan, 2017), y que consiste básicamente en ir diferenciando las diferentes partes de la situación y comprendiendo sus relaciones internas. Así pues, a lo largo de este continuo "simulación-autenticidad", que representa la articulación "universidad-escuela", tiene lugar continuamente un ciclo "prácticadescomposición de la práctica", que algunos autores han planteado en cuatro fases (Peercy y Troyan, 2017; McDonald et al., 2013).

Este modelo tiene el mérito de abordar el problema de la articulación universidadescuela. Sin embargo, presenta al mismo tiempo algunos retos. Por un lado, identificar las Core Practices es una tarea problemática (McDonald et al., 2013). En este sentido, para empezar, hay la cuestión de definir qué es una "práctica" y cómo se distingue de una competencia, un procedimiento o una técnica. Una vez esta cuestión pudiera ser superada, está todavía el problema de decidir qué prácticas son centrales y cuáles no lo son. Por otro lado, el modelo se centra en la construcción de conocimiento situacional, pero el conocimiento proposicional y la investigación académica parecen no tener ningún rol relevante en el currículum. Esto levanta dudas sobre si la CPTE puede suponer realmente una solución al problema teoría-práctica; es cierto que parece solucionar la articulación universidad-escuela, pero lo hace a base de "practicalizar" la universidad, convirtiéndola en un espacio de simulación de prácticas y de construcción de repertorio a partir de las mismas. La investigación académica queda fuera del currículum, aunque se usa, por parte de los académicos y formadores, para investigar las Core Practices que, estas sí, constituyen el currículum (McDonald et al., 2013).

\section{DISCUSIÓN}

En este artículo he intentado mostrar que el problema de la articulación entre la teoría y la práctica admite distintas definiciones desde un punto de vista psicológico, y que estas definiciones tienen importantes repercusiones en los modelos de formación inicial del profesorado.

Desde un punto de vista psicológico, la cuestión no está resuelta. Por un lado, es difícil asumir una versión fuerte de la cognición situada, en el sentido de negar la posibilidad de transferencia, porque existe evidencia que indica que la transferencia entre contextos es a veces posible. Sin embargo, la hipótesis de que el contexto concreto penetra en la cognición y la transforma (o, en otros términos, que la cognición se distribuye en el contexto) es probablemente correcta. Por otro lado, el cuarto supuesto de la racionalidad técnica -según el cual, el conocimiento proposicional explícito guía la acción- es probablemente falso. Sin embargo, no está claro por qué razón es falso; si es por la división computacional entre lo implícito y lo explícito, o si lo es por la diferencia entre el conocimiento situacional y el proposicional. Los modelos de doble proceso son plausibles, aunque, en su versión fuerte, 
son poco conciliables con algunas observaciones de la cognición situada. Sin embargo, la hipótesis de la redescripción representacional, que implica una versión menos fuerte de estos modelos, levanta algunas dudas desde el punto de vista de la evidencia. Por su parte, la hipótesis basada en la distinción entre lo proposicional y lo situacional parece más conciliable con las observaciones de la cognición situada. Esta hipótesis postula que el impacto del conocimiento proposicional en la acción es difuso, ya que tiene lugar a través de su influencia en la construcción del conocimiento situacional. Sin embargo, no está claro qué formas toma esta influencia -y, por lo tanto, cuando se puede considerar que una proposición teórica interviene en una acción concreta- y tampoco está claro qué condiciones permiten que una proposición explícita determinada influya en la construcción de conocimiento situacional.

El problema de la articulación entre teoría y práctica es un reto para cualquier propuesta de formación inicial del profesorado. En este artículo he intentado mostrar que las respuestas que se dan a este reto dependen de cómo se define el problema. En este sentido, los modelos examinados aquí proponen varias alternativas. La negación de la transferencia da lugar a la SBTE y a la RBTE, aunque con soluciones opuestas; la negación de la idea que las proposiciones explícitas guían la acción da lugar a la RTE y a la CPTE, aunque la primera pone el énfasis en el eje implícito-explícito, y la segunda lo pone en el eje proposicional-situacional. Todos los modelos, sin embargo, siguen sin resolver realmente la cuestión. La RBTE mantiene el equilibrio entre teoría y práctica, pero en este modelo se continúa observando una discontinuidad entre ambas. Las otras tres propuestas, la SBTE, la RTE y la CPTE solucionan esta discontinuidad eliminando una parte del problema: la teoría (el conocimiento proposicional explícito y general, generado mediante investigación académica). La SBTE lo hace basándose en una negación de la posibilidad de transferencia. La RTE intenta esta articulación a partir de la redescripción representacional, pero acaba proponiendo que dicha redescripción termine en conocimiento de tipo local (no teórico). La CPTE se centra en construir conocimiento situacional, prácticamente desterrando del currículum el conocimiento proposicional teórico.

Es evidente que hace falta avanzar hacia una mejor comprensión del problema a nivel psicológico, lo cual permitiría recomendaciones más certeras sobre la dirección que podrían tomar los modelos de formación inicial del profesorado. En el presente estado de la cuestión, sin embargo, me parece que hay algunas orientaciones que pueden ser asumidas para avanzar. La primera, y quizás la más importante, es mantener cierta prudencia con relación a decisiones que, de acuerdo con la comprensión actual del fenómeno, podrían ser un poco arriesgadas. Así, por un lado, la negación de la transferencia es un supuesto en discusión, como también lo es la idea que las proposiciones teóricas (generales y explícitas) no intervienen en la toma de decisiones. En consecuencia, no parece prudente que el currículum de la formación del profesorado renuncie a incorporar la investigación académica y el conocimiento teórico, ni que se desequilibre la formación del profesorado excesivamente hacia el contexto de práctica. A partir de un cierto equilibrio en estos aspectos, la evidencia indica que el supuesto de la transferencia (tal como lo asumía la racionalidad técnica) debe ser, como mínimo, matizado. En este sentido, la propuesta de la RBTE de generar un solo contexto de práctica (multi-institucional y complejo) que incluya investigación académica y práctica docente puede permitir abordar la cuestión de la transferencia manteniendo el equilibrio teoría-práctica y universidad-escuela. En esta dirección, la creación de "terceros espacios", propuestos sobre todo desde la SBTE, 
puede funcionar como primer paso, como germen, para avanzar hacia la fusión de los otros dos espacios (universidad y escuela) en uno solo. La evidencia indica también que el supuesto según el cual las proposiciones teóricas guían la acción debe ser igualmente, como mínimo, matizado. Desde este punto de vista, tanto la RTE como la CBTE proponen que, en relación con esta cuestión, los procesos de reflexión (explícitos y conscientes) sobre la práctica son necesarios. Estos procesos se plantean de forma diferente en los dos modelos: en la RTE, se basan en la explicitación, ya que el énfasis explicativo se pone en el eje implícito-explícito; en la CBTE, se basan en la descomposición de la práctica, ya que el énfasis explicativo se pone en el eje situacional-proposicional. El estado de la cuestión actual no permite hacer una afirmación fuerte sobre qué alternativa explicativa parece ser más correcta. Sin embargo, parecería recomendable que, por un lado, estos procesos de reflexión no renunciaran a las proposiciones teóricas, y, por otro lado, que el énfasis en dichos procesos no desequilibrara excesivamente el currículum al punto de perjudicar la formación científica en educación.

En este artículo se ha propuesto que añadir el estrato psicológico a la comprensión de los modelos de formación del profesorado, especialmente con relación al problema teoría-práctica, puede añadir profundidad a dicha comprensión y señalar vías de mejora. En este sentido, de acuerdo con la comprensión psicológica actual de la cuestión, se puede sugerir tres aspectos hacia los cuales la formación inicial del profesorado podría avanzar: 1) un equilibrio, en el currículum, entre investigación académica y práctica docente; 2) un equilibrio entre formación en la universidad y formación en la escuela; 3) la generación de un solo contexto, y una identidad compartida, que incluya universidad y escuela, investigación y práctica docente; 4) un énfasis importante en los procesos de reflexión sobre la práctica, sin renunciar al rol de las proposiciones teóricas en estos procesos.

\section{REFERENCIAS BIBLIOGRÁFICAS}

Admiraal, W., Buijs, M., Claessens, W., Honing, T. y Karkdijk, J. (2017). Linking theory and practice: Teacher research in history and geography classrooms. Educational Action Research, 25(2), 316-331. doi: 10.1080/09650792.2016.1152904.

Aspfors, J. y Eklund, G. (2017, publicado online). Explicit and implicit perspectives on researchbased teacher education: Newly qualified teachers' experiences in Finland. Journal of Education for Teaching. doi: 10.1080/02607476.2017.1297042.

Clarà, M. (2014). What is reflection? Looking for clarity in an ambigious notion. Journal of Teacher Education, 66(3) 261-271. doi: 10.1177/0022487114552028.

(2015). Representation and emotion causation: a cultural psychology approach. Culture \& Psychology, (21), 37-58. doi: 10.1177/1354067X14568687.

Buitink, J. (2009). What and how do student teachers learn during school-based teacher education. Teaching and Teacher Education, 25(1), 118-127. doi: 10.1016/j.tate.2008.07.009.

Cochran-Smith, M. y Lytle, S.L. (1999). Relationships of knowledge and practice: teacher learning in communities. Review of Research in Education, 24, 249-305. doi: 10.3102/0091732X024001249.

Dewey, J. (1989). Cómo pensamos. Nueva exposición de la relación entre pensamiento reflexivo y proceso educativo. Barcelona: Paidós.

Duit, R. y Treagust, D.F. (2003). Conceptual change: A powerful framework for improving science teaching and learning. International Journal of Science Education, 25(6), 671-688. doi: 10.1080/09500690305016.

Epstein, S. (2003). Cognitive-experiential self-theory of personality. En T. Millon, \& M. J. Lerner 
(Eds.), Personality and social psychology: Vol. 5. Comprehensive handbook of psychology (pp. 159-184). Hoboken, NJ: Wiley \& Sons.

Fodor, J. A. (1983). The modularity of mind. An essay on faculty psychology. Cambridge, MA: The MIT Press.

Forzani,F.M. (2014). Understanding "core practices" and "practice-based" teacher education: Learning from the past. Journal of Teacher Education, 65(4), 357-368. doi: 10.1177/0022487114533800.

Grossman, P., Compton, C., Igra, D., Ronfeldt, M., Shahan, E. y Williamson, P. (2009). Teaching practice: A cross-professional perspective. Teachers College Record, 111(9), 2055-2100.

Hansén, S-E., Forsman, L., Aspfors, J. y Bendtsen, M. (2012). Visions for teacher education. Experiences from Finland. Acta Didactica Norge, 6(1), Art. 9.

Hutchins, E. (1995). Cognition in the wild. Cambridge: MIT Press.

Jackson, A. y Burch, J. (2018, publicado online). New directions for teacher education: Investigating school/university partnership in an increasingly school-based context. Professional Development in Education. doi: 10.1080/19415257.2018.1449002.

Johnson-Laird, P. N. (1983). Mental Models. Towards a Cognitive Science of Language, Inference, and Consciousness. Cambridge: Cambridge University Press.

Kahneman, D. (2003). A perspective on judgment and choice. Mapping bounded rationality. American Psychologist, 58(9), 697-720. doi: 10.1037/0003-066X.58.9.697.

Karmiloff-Smith, A. (1992). Beyond modularity. A developmental perspective on cognitive science. Cambridge, MA: The MIT Press.

Klein, G. (2008). Naturalistic decision making. Human Factors, 50(3), 456-460. doi: $10.1518 / 001872008 X 288385$.

Klein, G. (2015). A naturalistic decision making perspective on studying intuitive decision making. Journal of Applied Research in Memory and Cognition, 4, 164-168. doi: 10.1016/j. jarmac.2015.07.001.

Korthagen, F. (2017). Inconvenient truths about teacher learning: Towards professional development 3.0. Teachers and teaching, 23(4), 387-405.

Korthagen, F. A. J. (2001). Linking practice and theory. The pedagogy of realistic teacher education. Mahwah: Lawrence Erlbaum. . (2010a). How teacher education can make a difference. Journal of Education for Teaching: International Research and Pedagogy, 36(4), 407-423. doi: 10.1080/02607476.2010.513854.

. (2010b). Situated learning theory and the pedagogy of teacher education: Towards an integrative view of teacher behavior and teacher learning. Teaching and Teacher Education, 26, 98-106. doi: 10.1016/j.tate.2009.05.001.

Korthagen, F.A.J. y Kessels, J.P.A.M. (1999). Linking theory and practice: Changing the pedagogy of teacher education. Educational Researcher, 28(4), 4-17.

Lave, J. (1991). La cognición en la práctica. Barcelona: Paidós.

Lawson, H. Norwich, B. y Nash, T. (2013). What trainees in England learn about teaching pupils with special educational needs/disabilities in their school-based work: The contribution of planned activities in one-year initial training courses. European Journal of Special Needs Education. 28(2), 136-155. doi: 10.1080/08856257.2013.778115.

Lillejord, S. y Borte, K. (2016). Partnership in teacher education -a research mapping. European Journal of Teacher Education, 39(5), 550-563. doi: 10.1080/02619768.2016.1252911.

Maaranen, K. y Krokfors, L. (2008). Researching pupils, schools and oneself. Teachers as integrators of theory and practice in initial teacher education. Journal of Education for Teaching, 34(3), 207-222. doi: 10.1080/02607470802213825.

McDonald, M., Kazemi, E. y Kavanagh, S.S. (2013). Core practices and pedagogies of teacher education: A call for a common language and collective activity. Journal of Teacher Education, 64(5), 378-386. doi: 10.1177/0022487113493807.

Nardi, B.A. (1996). Studying context: A comparison of activity theory, situated action models, and 
Estudios Pedagógicos XLV, N²: 179-195, 2019

EL PROBLEMA TEORÍA-PRÁCTICA EN LOS MODELOS DE FORMACIÓN DEL PROFESORADO: UNA MIRADA PSICOLÓGICA

distributed cognition. En B.A. Nardi (Ed.), Context and consciousness: Activity theory and human-computer interaction (pp. 69-102). Cambridge: MIT Press.

Nunes-Carraher, T., Carraher, D.W. y Schliemann, A.D. (1985). Mathematics in the streets and in schools. The British Psychological Society, 3, 21-29.

Park, H., Hewson, P.W., Lemberger, J. y Marion, R.D. (2010). The interactions of conceptions of teaching science and environmental factors to produce praxis in three novice teachers of science. Research in Science Education, 40, 717-741. doi: 10.1007/s11165-009-9141-1.

Peercy, M.M. y Troyan, F.J. (2017). Making transparent the challenges of developing a practice-based pedagogy of teacher education. Teaching and Teacher Education, 61, 26-36. doi: 10.1016/j. tate.2016.10.005.

Pozo, J. I., Scheuer, N., Mateos, M. y Pérez Echevarría, M. P. (2006). Las teorías implícitas sobre el aprendizaje y la enseñanza. En I. Pozo, N. Scheuer, M. P. Pérez Echevarría, M. Mateos, E. Martín y M. de la Cruz (Eds.), Nuevas formas de pensar la enseñanza y el aprendizaje. Las concepciones de profesores y alumnos (pp. 29-54). Barcelona: Graó.

Pozo, J. I. (2007). Ni cambio ni conceptual: La reconstrucción del conocimiento científico como un cambio representacional. En J.I. Pozo y F. Flores (Eds.), Cambio conceptual y representacional en la enseñanza de la ciencia (pp. 73-90). Boadilla del Monte: A. Machado Libros.

(2017). Aprender más allá del cuerpo: De las representaciones encarnadas a la explicitación mediada por representaciones externas. Infancia y Aprendizaje, 40(2), 219-276. doi: 10.1080/02103702.2017.1306942.

Pozo, J. I., Martín, E., Perez-Echeverría, M.P., Scheuer, N., Mateos, M. y de la Cruz, M. (2010). Ni contigo ni sin ti... Las relaciones entre cognición y acción en la práctica educativa. Infancia y Aprendizaje, 33(2), 179-184. doi: 10.1174/021037010791114580.

Puustinen, M., Säntti, J., Koski, A. y Tammi, T. (2018). Teaching: A practical or research-based profession? Teacher candidates' approaches to research-based teacher education. Teaching and Teacher Education, 74, 170-179. doi: 10.1016/j.tate.2018.05.004.

Schön, D. A. (1987). La formación de profesionales reflexivos. Hacia un nuevo diseño de la enseñanza y el aprendizaje en las profesiones (Trad. de L. Montero y J. M. Vez). Barcelona: Ediciones Paidós Ibérica, S.A.

Schön, D. A. (1998). El profesional reflexivo. Cómo piensan los profesionales cuando actúan (Trad. de J. Bayo). Barcelona: Ediciones Paidós Ibérica, S.A.

Scribner, S. (1984). Studying working intelligence. En B. Rogoff \& J. Lave (Eds.), Everyday cognition: Its development in social context (pp. 9-40). Cambridge: Harvard University Press.

Smith, K. (2015). The role of research in teacher education. Research in Teacher Education, 5, 43-46.

Stones, E. (1992). Quality teaching: A sample of cases. London and New York: Routledge.

ten Dam, G.T. y Blon, S. (2006). Learning through participation. The potential of school-based teacher education for developing a professional identity. Teaching and Teacher Education, 22, 647-660. doi: 10.1016/j.tate.2006.03.003.

Tillema, H.H. y Knoll, W.E. (1997). Promoting student teacher learning through conceptual change or direct instruction. Teaching and Teacher Education, 13(6), 579-595.

Tirri, K. (2014). The last 40 years in Finnish teacher education. Journal of Education for Teaching, 40 (5), 600-609. doi: 10.1080/02607476.2014.956545.

Toom, A., Krokfors, L., Kynäslahti, H., Stenberg, K., Maaranen, K., Jyrhämä, R., Byman, R. y Kansanen, P. (2008). Exploring the essential characteristics of research-based teacher education from the viewpoint of teacher educators. En B. Astrand, E. Eisenschmidt, B. Hudson, M. Lampere y P.Zgaga (Editors), Proceedings of Second Annual Teacher Education Policy in Europe Network (TEPE) Conference: Mapping the landscapte and looking to the future (pp.166179). Ljubljuana, Slovenia: TEPE Conference.

Toom, A., Kynäslahti, H., Krokfors, L., Jyrhämä, R, Byman, R., Stenberg, K., Maaranen, K. y Kansanen, P. (2010). Experiences of a research-based approach to teacher education: suggestions 
for future policies. European Journal of Education, 45(2), 331-344. doi: 10.1111/j.14653435.2010.01432.x.

van Velzen, C. y Volman, M. (2009). The activities of a school-based teacher educator: a theoretical and empirical exploration. European Journal of Teacher Education, 32(4), 345-367. doi: 10.1080/02619760903005831.

Zeichner, K. (2010). Rethinking the connections between campus courses and field experiences in college- and university-based teacher education. Journal of Teacher Education, 61(1-2), 89-99. doi: $10.1177 / 0022487109347671$.

. (2012). The turn once again toward practice-based teacher education. Journal of Teacher Education, 63(5), 376-382. doi: 10.1177/0022487112445789.

. (2014). The struggle for the soul of teaching and teacher education in the USA. Journal of Education for Teaching, 40(5), 551-568. doi: 10.1080/02607476.2014.956544. 
